\title{
Paraneoplastic pemphigus
}

INSERM

\section{Source}

INSERM. (1999). Orphanet: an online rare disease and orphan drug data base.

Paraneoplastic pemphigus. ORPHA:63455

Pemphigus is a group of chronic autoimmune skin diseases characterised by blisters formation on the outer layer of the skin and the mucous membranes. Three clinical forms have been characterised, of which paraneoplastic pemphigus is extremely rare. 